\title{
Epidemiologic Trends and Aspects of Severe Fever with Thrombocytopenia Syndrome Outbreaks in Korea and Japan, 2013 2017
}

\author{
Won-Chang Lee, DVM, MPH, Ph.D. ${ }^{1}$, Seung-Yong Park, DVM, M.S., Ph.D. ${ }^{1}$,
} Nong-Hoon Choe, DVM, M.S., Ph.D. ${ }^{1}$, Young Hwan Kwon, M.D. ${ }^{2}$

${ }^{1}$ College of Veterinary Medicine, Konkuk University, Seoul, ${ }^{2}$ Department of Internal Medicine, Aeromedical Center of Korean Air, Seoul, Korea

Received: July 8, 2020

Revised: July 13, 2020

Accepted: July 13, 2020

Corresponding Author

Young Hwan Kwon

Aeromedical Center, Korean Air, 260 Haneul-

gil, Gangseo-gu, Seoul 07505, Korea

Tel: $+82-2-2656-7150$

Fax: +82-2-2656-8848

E-mail: kumcim@hanmail.net

ORCID

https://orcid.org/0000-0001-9901-7280

\begin{abstract}
This study focuses on the comparative and quantitative analysis of the epidemiologic trends and aspects of severe fever with thrombocytopenia syndrome (SFTS) outbreaks between Korea and Japan from 2013 to 2017. The following factors were analyzed; cumulative incidence rate (CIR), cases-fatality rate (CFR), and the epidemic aspects, including cases related to gender, male-to-female morbidity ratio (MFMR), age, seasonal, and geographical distributions. We observed 607 SFTS cases with CIR in Korea during the period 2013 to 2017 were as 0.24 per 100,000 populations and with a 127 fatal-cases (F.C.s), corresponding to a CFR of $20.9 \%$, respectively. During the same period in Japan, 319 SFTS cases with a CIR of 0.05 and with 60 F.C.s to a CFR of $18.8 \%$ observed. When compared, the CIR of SFTS in Korea was significantly higher than in Japan $(P<0.01)$, but there were no significant differences levels of the CFR and MFMR between Korea and Japan. Also, a higher incidence of SFTS was observed in people aged over 50-years or elders in Korea and those of 60-years or elders in Japan $(P<0.01)$. The seasonal distribution of SFTS outbreak cases showed that the incidence in summer through autumn in Korea $(92.4 \%$ of total cases) was higher than in Japan (65.2\%), while the outbreaks of SFTS in spring was much higher in Japan (31.0\%) than in Korea (7.4\%), $(P<0.01)$. The regional distribution revealed no significant difference between the eastern area $(44.8 \%)$ and the western area (46.8\%) of the Korean peninsula except Jeju-island (8.4\%). However, in Japan, the incidence only occurred in Chubu-Kinki-Chugoku (30.3\%), Shikoku (25.7\%), Kyushu (42.6\%) and Okinawa (0.3\%), which are the western and southern areas of Japan. These differences in SFTS occurrence may reflect the influences of vector/ hosts, climate, and geographical and cultural characteristics between the two countries.
\end{abstract}

Keywords: Phlebovirus, Epidemiology, Risk factors, Republic of Korea, Japan

\section{INTRODUCTION}

Severe fever with thrombocytopenia syndrome (SFTS) is an emerging tick-borne hemorrhagic fever of the zoonotic disease in East Asia caused by the SFTS virus (SFTSV), a phlebovirus in the family Bunyaviridae. SFTS had been found for the first time in 2010 in China [1], and in 2013, it was also reported in Korea [2,3], and in 
Japan [4]. Thereafter in Korea, SFTS was legally classified as a type IV notifiable disease by the Communicable Disease Prevention Act of the Korea Center for Disease Control and Prevention (KCDC) in 2013 [5,6]. In Japan, SFTS was designated as category IV Infectious Diseases in 2013, requiring notification to the designated public health center $[4,7,8]$. The significant clinical symptoms of SFTS are fever, vomiting, diarrhea, multiple organ failure, thrombocytopenia, leucopenia, and elevated liver enzyme levels and has a fatality rate of $12 \%$ and as high as $30 \%$ in some areas [1-4,7-9]. The SFTSV has been detected in the Haemaphysalis longicornis, including several tick species, which has implicated as a vector of the virus [1]. $H$. longicornis ticks widely inhabit the Korean Peninsula and Japan [1,10-12], and seroconversion and viremia of SFTS have been demonstrated in domestic animals such as goats, sheep, cattle, pigs, and dogs; these animals have been implicated as an intermediate host in SFTSVendemic areas [2,4]. Recently, the reports of SFTS with SFTSV state that migratory birds, which breed in and migrate through China, Korea, and Japan, increase the potential to disperse $H$. longicornis-borne SFTS in these countries. The migratory bird routes and the distribution of $H$. longicornis are concurrent with the occurrence of SFTSV [3,9,13-17].

In this comparative descriptive study, understanding the epidemiologic trends and aspects and significant risk factors of SFTS outbreaks in Korea and Japan provides data necessary for performing a risk assessment and established effective health policies.

\section{MATERIALS AND METHODS}

We analyzed the following factors; cumulative incidence

Table 1. Comparative observation of epidemiological aspects and risk factors of SFTS between Korea and Japan, 2013 2017

\begin{tabular}{|c|c|c|c|c|}
\hline \multirow{2}{*}{ Item } & \multicolumn{2}{|c|}{ Korea } & \multicolumn{2}{|c|}{ Japan } \\
\hline & Cases (\%) & $95 \% \mathrm{Cl}$ & Cases (\%) & $95 \% \mathrm{Cl}$ \\
\hline Total cases & 607 & & 319 & \\
\hline $\mathrm{CIR} / 100,000$ & $0.24^{* *}$ & $0.22 \sim 0.26$ & 0.05 & $0.04 \sim 0.06$ \\
\hline Fatal-cases & 127 & & 60 & \\
\hline CFR/100 & 20.9 & $17.7 \sim 24.1$ & 18.8 & $14.5 \sim 23.1$ \\
\hline \multicolumn{5}{|l|}{ Gender } \\
\hline Male & 309 (50.9) & 46.9 54.9 & $152(47.6)$ & $42.1 \sim 53.3$ \\
\hline Female & $298(49.1)$ & $45.1 \sim 53.1$ & $167(52.4)$ & $46.9 \sim 57.9$ \\
\hline Total & 607 & & 319 & \\
\hline MFMR & 1.04 & & 0.91 & \\
\hline \multicolumn{5}{|l|}{ Age-specific } \\
\hline$<19$ & $4(0.7)$ & - & $2(0.6)$ & - \\
\hline 20 29 & $14(2.3)$ & $1.1 \sim 3.5$ & $1(0.3)$ & - \\
\hline 30 39 & $11(1.8)$ & $0.7 \sim 2.9$ & $6(1.9)$ & $0.7 \sim 3.2$ \\
\hline $40 \sim 49$ & $30(4.9)^{*}$ & $3.2 \sim 6.6$ & $5(1.6)$ & $0.2 \sim 3.0$ \\
\hline $50 \sim 59$ & $132(21.7)^{* *}$ & 18.4 25.0 & $20(6.3)$ & $3.6 \sim 9.0$ \\
\hline $60 \sim 69$ & $160(26.4)$ & $22.3 \sim 30.5$ & $88(27.6)$ & $22.7 \sim 32.5$ \\
\hline$>70$ & $256(42.2)$ & $37.3 \sim 47.1$ & $197(61.8)^{* *}$ & $56.5 \sim 67.1$ \\
\hline Total & 607 & & 319 & \\
\hline$P$-value & $<0.01$ & & $<0.01$ & \\
\hline \multicolumn{5}{|l|}{ Seasonality } \\
\hline Spring & $45(7.4)$ & $5.3 \sim 9.5$ & $99(31.0)^{* *}$ & $25.9 \sim 36.1$ \\
\hline Summer & $261(43.0)$ & $38.7 \sim 47.3$ & $148(46.4)$ & $40.9 \sim 51.9$ \\
\hline Autumn & $300(49.4)^{* *}$ & $45.4 \sim 53.4$ & 60 (18.8) & 14.5 23.1 \\
\hline Winter & $1(0.2)$ & - & $12(3.8)^{* *}$ & $1.7 \sim 5.9$ \\
\hline Total & 607 & & 319 & \\
\hline$P$-value & $<0.01$ & & $<0.01$ & \\
\hline
\end{tabular}

Remarks: CFR: case-fatality rate in \%, MFMR: male-female-morbidity ratio. 95\%, Cl: confident interval of 95\%. Statistically difference between the risk factors were determined using the Chi-square test, set at ${ }^{*} P<0.05$ and ${ }^{* *} P<0.01$. 
rate (CIR), cases-fatality rate (CFR), and the epidemiological aspects, including cases related to gender, male-tofemale morbidity ratio (MFMR), age, seasonal, and geographical distributions of SFTS cases during the period 2013 to 2017.

The raw data on conformed SFTS cases in Korea obtained from the National Notified Disease Surveillance System (Website) of the KCDC; an agency of the Ministry of Health and Welfare from 2013 to 2017 [6]. Data on reported SFTS cases in Japan obtained from the $\mathrm{Na}-$ tional Institute Infectious Diseases (NIID) in Japan from 2013 to $2017[7,8]$. In this study, the CIR of SFTS cases per 100,000 populations estimate by the criteria that the World Health Organization (WHO) established, and upper and lower limits of the 95\% confidence interval (95\% CI) were calculated. Statistical significant differences between the epidemiological aspects and risk factors were determined using Pearson's chi-square test or paired t-test. All data analyses performed in Excel 2010 (Microsoft Corp., Redmond, WA, USA). Results were considered statistically significant for a $P$-value of less than $<0.05$.

\section{RESULTS}

As shown in Table 1, comparative observation of the CIR (nationwide) of SFTS cases in Korea and Japan measured from 2013 to 2017 and compared. In Korea, there were 607 SFTS infections with a CIR of 0.24 per 100,000 populations (95\% CI: 0.22 0.26), and 127 FCs with a CFR of $20.9 \%$, respectively. During the same period in Japan, the CIR, FCs, and CFR were 0.05 per 100,000 (95\% CI: 0.04 0.06), 60 cases and 18.8\% (95\% CI: 14.1 23.1), respectively. The CIR of Korea was much higher than in

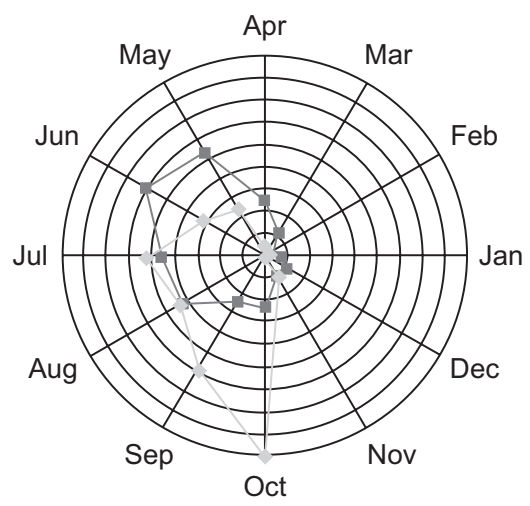

Fig. 1. Trends in distribution patterns of SFTS outbreaks by monthly in Korea and Japan, 2013 2017.
Japan's $(P<0.01)$, but the CFR was not significantly different. The effects of SFTS by gender and MFMR values in both Korea (1.04) and Japan (0.92) that similarly observed between men and women (Table 1).

The distribution rates of SFTS cases according to agespecific adjusted groups in Korea were as follows: for the age groups of under 19, 20 29, 30 39, 40 49, 50 59, $60 \sim 69$, and over 70 years old the percentages were $0.7 \%$, $2.3 \%, 1.8 \%, 4.9 \%, 21.7 \%, 26.4 \%$, and $42.2 \%$, respectively, $(P<0.01)$. In Japan, the proportions for the same age-specific adjusted groups were $0.6 \%, 0.3 \%, 1.9 \%, 1.6 \%, 6.3 \%$, $27.6 \%$, and $42.2 \%$, respectively, $(P<0.01)$. Moreover, the distribution of SFTS cases by the age-adjusted group were similar between Korea and Japan, with more than $90.3 \%$ of the cases occurring in individuals more than 50 years of age in Korea, but 60 years older (89.4\%) in Japan (Table 1).

The epidemiological relative risk factors of SFTS according to the season and environment of each individual analyzed. The seasonal distribution of the SFTS patients throughout the year revealed that outbreaks in summer and autumn (92.4\% of total cases) were more frequent in Korea than in Japan $(65.2 \%),(P<0.01)$. In Korea, the outbreaks increased sharply in May, the highest in October ( $28.8 \%$ of total cases), and suddenly began to decline in November, when the cold season began. However, in Japan, the SFTS outbreaks increased in April, peaked in May (22.6\% of total outbreaks) through October, and tended to decline in November (Fig. 1).

Table 2 showed the geographical distribution of SFTS outbreaks of Korea and Japan. The regional distribution

Table 2. Geographical distribution of SFTS cases in Korea and Japan, 2013 2017

\begin{tabular}{llcllc}
\hline \multirow{2}{*}{ Area } & \multicolumn{2}{c}{ Korea } & & \multicolumn{2}{c}{ Japan } \\
\cline { 2 - 3 } \cline { 5 - 6 } & \multicolumn{1}{c}{ Districts } & Cases (\%) & & Districts & Cases (\%) \\
\hline \multirow{2}{*}{ Eastern } & Gangwon & $90(14.8)$ & & Hokkaido & 0 \\
& Kyeongsang & $182(30.0)$ & & Kanto & 0 \\
& Seoul City & $30(4.9)$ & & Chubu & $10(3.1)$ \\
& Gyeonggi & $111(18.3)$ & & Kinki & $17(5.3)$ \\
& Chungcheong & $83(13.7)$ & & Chugoku & $70(21.9)$ \\
Southern & Chula & $60(9.9)$ & & \\
& Jeju-Do & $51(8.4)$ & & Shikoku & $82(25.7)$ \\
& & & & Kyushu & $136(42.6)$ \\
Unknown & & & & Okinawa & $1(0.3)$ \\
Total & & 607 & & $3(0.9)$ \\
\hline
\end{tabular}


revealed no significant difference between the eastern area $(44.8 \%)$ and the western area (46.8\%) of the Korean peninsula except Jeju-island (8.4\%). However, in Japan, the incidence only occurred in Chubu-Kinki-Chugoku (30.3\%), Shikoku (25.7\%), Kyushu (42.6\%), and Okinawa $(0.3 \%)$, which are the western and southern areas of Japan.

\section{DISCUSSION}

SFTS was the first discovered in rural areas of middleeastern China in 2009 as an emerging tick-borne zoonosis affecting hundreds of cases reported in China each year [1,9-11]. Thereafter, in Japan, the first SFTS case was identified through the death of a female patient and a retrospective study on SFTS, 2012 [4]. In Korea, the first SFTS case reported a retrospectively identified fatal case in Korea, 2012 [2,3], and subsequently, SFTS was diagnosed in 607 patients, and there were 127 reported deaths during the period from 2013 to 2017 as showed in Table 1 . There was a CIR of 0.24 per 100,000 populations, and a CFR of $20.9 \%$ in Korea, and those of Japan (CIR 0.06 and CFR 18.6\%) were lower in respect to CIR than in Korea. As an emerging infectious disease, SFTS is considered an increasingly important public health threat because of its wide distribution and high CFR [1-5,7]. Regarding the effects of SFTS by MFMR observe in Korea and Japan, there was a statistically no significant difference. The highest incidence of infections was observed in individuals in the age of 50 59 in Korea $(P<0.01)$, and those aged 70 years or older in Japan $(P<0.01)$. The risky age group for SFTS infection was reported to be similar among Korea, Japan, and China, and over $90 \%$ of the cases occurred in the over-50-year-old age bracket clearly showing a higher incidence in the elderly. Because in these countries, most young people move to cities for work, and older people are left to work as farmers on their land $[1-3,5,11,12]$. Therefore, older people should be the primary target for preventive education of SFTS.

The distribution of SFTS cases throughout the year revealed that outbreaks frequently started from the end of spring to the middle of autumn (92.4\% of total cases) in Korea. On the other hand, a summary of outbreaks of SFTS with geography revealed significantly more outbreaks in the southern area of the Korean peninsula nearby Japan, as shown in Table 2 . These data strongly indicate that the outbreaks are influenced by the geographical and environmental conditions of the SFTS transmissible factor and that these differences in SFTS infections risk factors reflect the influence of vector/host, climate, and geographical and cultural characteristics between two countries. Also, the reports of SFTS with SFTSV, state that migratory birds, which breed in and migrate through China, Korea, and Japan, increase the potential of dispersing $H$. longicornis-borne SFTS in these countries. The migratory bird routes and the distribution of $H$. longicornis are concurrent with the occurrence of SFTSV, as recently published [3,15-19].

Finally, of all vector-borne zoonotic diseases, SFTSV infection is one of the most severe, as indicated by the CFRs of $20.9 \%$ in Korea and $18.6 \%$ in Japan, which signifies that it is higher fatal. Despite the widespread endemic of SFTS, the focal transmission of the disease allows for highly targeted prevention in high-risk areas. Transmission can be minimized through the health education of residents or travelers at high-risk areas, recommendation repellent- or pesticide-impregnated clothes, limited access to endemic areas, or other appropriate prophylactic measures [1-6,12,17-19].

In conclusion, SFTS is an emerging tick-borne zoonosis and a serious concern to public health measures of prevention and control. We observed that there is a similar pattern of epidemiological aspects of SFTS infections in Korea and Japan. These results underscore the continued emergency of SFTS and provide a basis for targeting prevention campaigns to the population with increasing incidence. For example, to prevent possible SFTS transmission from ticks, use insect repellents. Proper wear of clothing will also prevent transmission if you tuck your pants into your booth, shirt into your pants, and keep sleeves rolled down [1-8].

This study provides a retrospective and quantitative analysis of the epidemiological aspects and relative risk factors of SFTS infections in Korea and Japan. Moreover, we hope that this information will be a useful reference in the future study of SFTS.

\section{ACKNOWLEDGEMENTS}

The author thanks the Korea Center for Disease Control and Prevention (KCDC) and the National Institute of Infectious Diseases (NIID) in Japan. 


\section{CONFLICTS OF INTEREST}

No potential conflict of interest relevant to this article was reported.

\section{ORCID}

Won-Chang Lee, http://orcid.org/0000-0001-7163-5183

Seung-Yong Park, https://orcid.org/0000-0001-8104-8775

Nong-Hoon Choe, https://orcid.org/0000-0003-4727-7528

Young Hwan Kwon, http://orcid.org/0000-0001-9901-7280

\section{REFERENCES}

1. Yu XJ, Liang MF, Zhang SY, et al. Severe with thrombocytopenia associated with a novel bunya virus in China. New Engl J Med. 2011;364:1523-32.

2. Kim KH, Yi J, Kim G, Choi SJ, et al. Severe fever with thrombocytopenia syndrome, South Korea, 2012. Emerg Infect Dis. 2013;19:1892-4.

3. Park SW, Han MG, Yun SM, et al. Severer Fever with Thrombocytopenia Syndrome Virus, South Korea, 2013. Emerg Infect Dis. 2014;20:1880-2

4. Takahashi T, Maeda K, Suzuki T, et al. The first identification and retrospective study of severe fever with thrombocytopenia syndrome in Japan. J Infect Dis. 2014;209:81627.

5. Shin JS, Park JH, Kwon DH. Epidemiological and clinical characteristics of severe fever thrombocytopenia syndrome in the Republic of Korea. Public Health Weekly Report, KCDC. 2014;7:493-8.

6. Korea Center for Disease Control and Prevention. Severe fever with thrombocytopenia syndrome (SFTS), 2013-2017. Statistical System of the National Notifiable Disease Surveillance. Available from: https://is.cdc.go.kr/dstat/index. jsp.

7. National Institute of Infectious Diseases. Focus; Severe Fever with Thrombocytopenia Syndrome, Infectious Disease Weekly Report (IDWR), Dec. 28, 2016. Available from: https://www.niid.go.jp/niid/ja/idwr.html.

8. National Institute of Infectious Diseases. SFTS in Japan, as of February 2016, Infectious Agents Surveillance Report (IASR): 2016;37:39-40. Available from: https://www.niid. go.jp/niid/en/aboutniid-2/865-iasr/6339-tpc433.html.

9. Ding S, Niu G, XuX et al. Age is a critical risk factor for severe fever with thrombocytopenia syndrome. PLoS One. 2014 Nov 4;9(11):e111736. Available at http://www.plosone. org.

10. Chae JS, Yu DH, Shringi S, at al. Microbial pathogens in ticks, rodents, and a shrew in northern Gyeonggi-do near the DMZ, Korea. J Vet Sci. 2008;9:285-93.

11. Ding S, Yin H, Xu X, et al. A Cross-Section of Severe Fever with Thrombocytopenia Syndrome Virus Infection of Domestic Animals in Laizhou City, Shandong Province, China. Jpn J Infect Dis. 2014;67:1-4.

12. Liu K, Zhou H, Sun RX, et al. A national assessment of the epidemiology of severe fever with thrombocytopenia syndrome, China. Sci Rep. 2015;23:9679.

13. Lee KH, Medloke, JM, Heo ST. Severe Fever with Thrombocytopenia Syndrome Virus, Crimenan-Congo Hemorrhagic Fever Virus, and Migratory Birds. J Bacteriol Virol. 2013;43:235-43.

14. Yun Y, Heo ST, Kim G, et al. Phylogenetic analysis of severe fever with thrombocytopenia syndrome virus in South Korea and migratory bird routes between China, Korea, and Japan. Am J Trop Med Hyg. 2015;93:468-74.

15. Park SW, Song BG, Shin EH, et al. Prevalence of severe fever with thrombocytopenia syndrome virus in Haemaphysalis longicornis ticks in South Korea. Ticks Tick Borne Dis. 2014;5:975-7.

16. East Asia/Australasia Flyway-Birdlife International. Available at https://www.birdlife.org/sites/default/file/attachment/8_EAST_ASIA_Australia_Factsheet.pdf.

17. Choi CY, Kang CW, Yun YM, Nam HY. Can We Blame Migratory Birds for Transmission of the Emerging Severe Fever with Thrombocytopenia Syndrome Virus in East Asia? Am J. Trop. Med. Hyg. 2015;93:1391-2.

18. Yun SM, Song BG, Choi WY, et al. First Isolation of SFTS Virus from Haemaphysalis longicornis Ticks Collected in SFTS Outbreaks Areas in the Republic of Korea. VECTORBORNE AND ZOONOTIC DISEASES. 2016;16:66-70.

19. Yoshikawa T, Shimojima M, Fukshi S. Tani H, et al. Phylogenetic and Geographic Relationship With SFTS Virus in China, Korea, and Japan. J Infect Dis. 2015;212:889-98. 